\title{
Arabidopsis sculpt distinct root-associated microbiomes through the synthesis of secondary metabolites and defense signaling molecules
}

Enoch Narh Kudjordjie

Aarhus Universitet

Rumakanta Sapkota

Aarhus Universitet

Mogens Nicolaisen ( $\nabla$ mn@agro.au.dk)

Aarhus Universitet https://orcid.org/0000-0002-0407-2488

\section{Research}

Keywords: Plant microbiomes, defense signaling pathways, plant metabolites, glucosinolates, flavonoids, phytohormones

Posted Date: May 14th, 2020

DOl: https://doi.org/10.21203/rs.3.rs-27655/v1

License: (c) (i) This work is licensed under a Creative Commons Attribution 4.0 International License. Read Full License 


\section{Arabidopsis sculpt distinct root-associated microbiomes through the synthesis of}

2 secondary metabolites and defense signaling molecules

3 Enoch Narh Kudjordjie ${ }^{1}$, Rumakanta Sapkota ${ }^{1,2}$, Mogens Nicolaisen $^{1}$

$4 \quad{ }^{1}$ Aarhus University, Faculty of Technical Sciences, Department of Agroecology, Forsøgsvej 1,

5 DK4200, Slagelse Denmark.

$6 \quad{ }^{2}$ Current address: Aarhus University, Faculty of Technical Sciences, Department of Environmental

7 Science, Frederiksborgvej 399, 4000 Roskilde.

8 Corresponding author: mn@agro.au.dk

9

10

11

12

13

14

15

16

17

18

19

20

21

\section{Abstract}

\section{Background}

Plant mutants with alterations in specific biosynthetic or signaling pathways exhibit distinct biochemical or physiological traits and are, thus, suitable models for studying links between the plant and its associated microbiota. Here, we examined microbial community structures of a range of Arabidopsis thaliana mutants disrupted in metabolic pathways for the production of glucosinolates, flavonoids, or a number of defense signaling molecules. Arabidopsis mutants and their background wild types (controls) were grown in natural soil and maintained in a greenhouse for 4 weeks before collection of roots for microbiome analysis. We characterized bacterial and fungal communities using 16S rRNA and fungal ITS amplicon sequencing, respectively. 


\section{Results}

Our results showed that the Arabidopsis mutants had distinct microbial profiles compared to control plants. The relative abundances of the bacterial classes Actinobacteria, Thermoleophilia and Verrucomicrobiae, and the fungal classes Eurotiomycetes and Sordariomycetes were the most affected when comparing mutants and their wild types. At the genus level, the bacterial taxa Azospirillum, Fluviicola, and Flavobacterium were significantly enriched in most glucosinolate, flavonoid and signaling mutants while the fungal taxa Sporobolomyces and Emericellopsis were enriched in several glucosinolate and defense signaling mutants.

\section{Conclusion}

By using different Arabidopsis mutants and their background controls, we showed that plant secondary metabolism and defense signaling molecules affect bacterial and fungal community structures. We conclude that disruption of pathways for secondary metabolite production or disruption of defense signaling pathways affected the innate mechanisms that modulate plant rootassociated microbiome assembly.

Keywords: Plant microbiomes, defense signaling pathways, plant metabolites, glucosinolates, flavonoids, phytohormones

\section{Introduction}

Plants interact with a vast diversity of microorganisms both above and belowground, and the outcomes of these interactions may be commensal, beneficial or detrimental to the plant. Essentially, the plant employs a range of strategies such as the action of preformed and/or induced chemical compounds in combination with the plant innate immune systems to sculpt the microbiota $(1,2)$. 
44 Plant secondary metabolites such as glucosinolates (GLS) and flavonoids (FLVs) have been widely studied for several microbiota-mediating and plant protective functions $(3,4)$. For instance, GLS from the roots of Brassica species were found to inhibit microbial pathogens including Pseudomonas syringae, Alternaria brassicicola, Gaeumannomyces graminis, Botrytis cinerea, Fusarium oxysporium and Peronospora parasitica $(5,6)$. The FLVs are well known for their chemoattractant and signaling function in legume-rhizobia interactions resulting in $\mathrm{N}$-fixation $(7,8)$. FLVs also possess antimicrobial activity used in defense against pathogens. They are involved in plantmycorrhizal associations, and act as enhancers of bacterial growth rates $(7,8)$. The activation of the innate plant immune system is inevitable due to the constant interaction between plants and microbes (1). The plant immune system, which is highly networked with other defense repertoires, is under constant activation by perception of microbial associated molecular patterns resulting in a broad spectrum of inducible defense responses (9). Plant hormones serve as signaling molecules in regulating the innate immune network. Phytohormones including salicylic acid (SA), jasmonic acid (JA), ethylene (ET) and abscisic acid (ABA) act as molecular switches in stimulating inducible defense against biotic and abiotic stresses (9). Owing to its robust and overarching activation of defense repertoires, the immune system is perceived to affect microbial community structures (10).

The biosynthetic pathways and genes involved in $\operatorname{GLS}(11,12,13)$, FLV $(14,15,16)$ and defense signaling $(17,18,19)$ are well described, and research is directed towards exploiting these pathways to study the links between plant gene functions and microbiome assemblage. Several wellcharacterized mutants of the model plant, Arabidopsis thaliana (hereafter Arabidopsis) have become quintessential for studying this relationship. For example, Badri et al. (20) reported effects on microbial communities of a mutation in a plant ATP transporter involved in exudation of plant secondary metabolites, and further concluded that individual plant genes are actively involved in the interaction with microbial communities. By using GLS (21), FLV (20) and benzoxazinoid (BX) 
mutants $(22,23)$, the influence of plant defensive secondary metabolites on the plant-associated microbiota has been demonstrated. For example, distinct microbiomes were observed in maize parental lines and their isogenic mutants (bx1, bx2 and bx6) carrying disruptions in genes encoding enzymes in different steps of the BX pathway (23). This study further demonstrated a gatekeeper role of BXs in modulating plant-associated microbiomes associated with plant roots. In other studies, the coumarin-impaired mutants, myb72-2, bglu42 and f6'h1 were used to demonstrate the impact of coumarin on microbial community assembly (24-26). In addition, studies have used Arabidopsis mutants to examine the influence of phytohormones on microbial community structures $(27,28)$.

Mechanistic processes at the rhizoplane, including the gating role of plant secondary metabolites (23) and defense signaling molecules (DSMs) (27,28), are controlling the assembly of host specificmicrobiomes. We hypothesized that mutations in pathways for the synthesis of certain secondary metabolites and DSMs disrupt the ability of the plant to sculpt its associated microbiome. While it is evident that single plant metabolites or DSMs affect host-associated microbiomes, studies that characterize the effect of a range of these chemical compounds on both bacterial and fungal microbiomes under similar and natural conditions are scarce. Such a comprehensive analysis using plant mutants could provide an in-depth and comparative understanding of the effects of different metabolites and DSMs on microbial structures. Thus, the aim of this study was to examine fungal and bacterial community structures of Arabidopsis mutants impaired in GLS, FLV and the DSMs SA, JA, ABA, ET and fatty acid biosynthesis. For comparison, we included the reference wild types used originally as backgrounds for generating the mutants. 


\section{Plant material}

We used 21 Arabidopsis mutants and their genetic background lines Col-0 and Ler-0 (Additional file 1: Table S1). The GLS (cyp79B2 and cyp79B3), FLVs (tt3, tt5) and jasmonic acid (dde2) mutants were kindly provided by Profs. Judith Bender (Brown University), Wendy Peer (University of Maryland) and Paul Staswich (University of Nebraska), respectively. The FLV mutants used in this study had a defective proanthocyanidin accumulation resulting in a transparent testa (tt) phenotype characterized by a yellowish or pale brown colour (29). Other Arabidopsis lines were supplied by the Nottingham Arabidopsis Stock Centre (NASC), UK.

\section{Experimental design}

Arabidopsis seeds were sown in pots $(8 \mathrm{~cm} \times 8 \mathrm{~cm} \times 6 \mathrm{~cm})$ with moistened field soils collected from a fallow field (Jyndevad Research station, Denmark). Each pot represents a biological replicate of individual genotypes and replicated 5 times for all genotypes. Seeds were stratified and pots completely randomized and maintained in a greenhouse under 2017 summer conditions (Additional file 1: Figure S1B). Seedlings were maintained by capillary watering and regular weed removal. Sampling was done after 4 weeks of plant growth where roots were harvested and the adhering soils gently shaken off. Roots and remaining attached soil were pooled and placed into $2 \mathrm{ml}$ collection tubes, frozen in liquid nitrogen and later stored in $-20{ }^{\circ} \mathrm{C}$ freezers. Subsequently, the samples were lyophilized and ground using a Geno/Grinder 2010 at a rate of $1500 \mathrm{rpm}$ with sterile metal balls (size $2.88 \mathrm{~mm}, 3 \mathrm{x}$ per sample) before DNA extraction. For detailed experimental procedure, see supplementary methods and Figure S1 (Additional file 1). 


\section{Sample processing, sequence analysis and statistics}

Sample DNA extraction and library preparation were essentially as previously described (23). Briefly, we extracted DNA using the PowerLyzer ${ }^{\mathrm{TM}}$ Power Soil ${ }^{\circledR}$ DNA Isolation Kit (Mo Bio Laboratories, Carlsbad, CA, USA). The bacterial primers S-D-Bact-0341-b-S-17, 5'CCTACGGGNGGCWGCAG-3' and S-D-Bact-0785-a-A-21, $5^{\prime}-$ GACTACHVGGGTATCTAATCC-3' (30) and the fungal primers fITS7, 5'GTGARTCATCGAATCTTTG-3’ and ITS4, 5’-TCCTCCGCTTATTGATATGC-3’ (31) were used to amplify the V3/V4 region of the bacterial $16 \mathrm{~S}$ rRNA and the fungal internal transcribed spacer 2 (ITS2) region, respectively. A dual indexing strategy was used and PCR conditions were as described (23). For details, see supplementary methods (Additional file 1). Samples were sequenced using the Illumina MiSeq platform at Eurofins MWG (Ebersberg, Germany). All the sequence files were deposited in the NCBI Sequence Read Archive (SRA) under the accession number PRJNA579829.

Sequence analysis for demultiplexing, chimera detection and removal, OTU picking, and OTU table creation were performed using vsearch version 2.6 (32), as described (23). Taxonomy assignment was carried out in QIIME version 1.9 (33). Downstream data exploration and visualization was performed in the R statistical package (34). Microbial community diversity estimations including alpha and beta diversities, species richness and dissimilarity were performed using the 'vegan' package (35) and phyloseq (36). A cutoff minimum of 500 reads for both bacteria and fungi, was used to remove samples with lower numbers of reads (Additional file 1: Figure S2). OTU tables were either transformed to relative abundance tables or rarified prior to alpha and beta diversity based calculations. To determine statistically significant differences in taxonomic profiles, we performed a multiple group analysis by comparing sequences assigned to different class level taxa (top 10) in mutants and their respective background controls (Col-0 or Ler-0) using the STAMP software v2.1.3 (37, 38). Genotypes were compared by ANOVA, followed by the Tukey-Kramer post hoc test ( $\mathrm{p}<$ 
0.05) using the Benjamini and Hochberg (BH) FDR for multiple comparisons. Taxa with small effect sizes were removed by filtering (effect size $=0.8$ ). Beta diversity based PERMANOVA for partitioning of variance was calculated using UniFrac weighted matrices for bacterial communities and Bray Curtis for fungal communities using the 'adonis' test. Adonis test was performed using genotypes with at least 3 replicates. Differential OTU analysis was performed using DESeq2 (version1.22.2) (39). Datasets partitioned for each mutant and reference control were subjected to differential analysis to determine the most differentially significant bacterial and fungal taxa.

\section{Results}

We studied microbiome composition in Arabidopsis plants carrying mutations in specific steps of GLS, FLV and defense signaling pathways (Additional file 1: Figures S3A- S3C and Table S1). To determine genotypic effects on bacterial and fungi community structures, we split the data for mutants and their background controls into these three groups.

\section{Species abundance differs between mutants and wild types}

Bacterial and fungal relative abundances were distinct in the GLS mutants and their wild type Col-0 (Additional file 1: Figures S4A, S4B). We found significant differences in the mean abundance of reads belonging to Actinobacteria, Thermoleophilia and Verrucomicrobiae. The comparison between the mutants cyp79B3, cyp79B2cyp79B3 and TU3, and the reference Col-0 showed the highest differences in mean proportions of reads in the respective taxa $(\mathrm{p}<0.001)$ (Fig. 1A). For fungi, Sordariomycetes was highly abundant across several mutants but also in Col-0, while Eurotiomycetes was enriched in mutants compared to Col-0 (Additional file 1: Figure S4B). By performing ANOVA and post hoc Tukey's test, we found significant differences in reads assigned to Eurotiomycetes (cyp79B3 and TU3: Col-0), Olpidiomycetes (pad2_1: Col-0) and Sordariomycetes (cyp79B3 and cyp79B2cyp79B3: Col-0 and gsm1-1: Col-0) (Fig. 1A). 
Class-level plots of bacterial and fungal relative abundances showed distinct microbial abundances in the FLV mutants and their controls (Additional file 1: Figures S4C-S4D). Multiple group analysis revealed significant differences of bacterial class assigned reads, with the strongest differences observed in Actinobacteria (Col-01:pap1-D), Phycisphaerae (Ler-0:tt7-7,), and Verrucomicrobiae (Ler-0:tt7-7, Ler-0:tt5 (Fig. 1B). The multiple test statistics for mean class level taxa comparison indicated significant differences in the mean of reads belonging to Eurotiomycetes (pap1-D:Col-0), Sordariomycetes (Col-0: pap1-D) and Tremellomycetes (Col-0: pap1-D) (Fig. 1B).

We detected shifts in the relative abundances of both bacteria and fungi at class-level in the defense signaling mutants (Additional file 1: Figures S4E- S4F). The bacterial class Verrucomicrobiae was the most significantly affected taxon detected in a comparison between Ler- 0 and aba3-2. Reads belonging to Alphaproteobacteria and Thermoleophilia were also significantly different, respectively in the aba3-2: Ler-0 and Col-0: npr1-2 comparison (Fig. 1C). For fungi, Sordariomycetes was significantly different between aba3-2 and Ler-0. In addition, we found significant differences in Olpidiomycetes abundance between Col-0 and pad3-1 and for Saccharomycetes between Ler-0 and aba3-2 (Fig. 1C).

Next, we performed differential analysis to determine microbial taxa that were significantly different between mutants and controls. A number of significantly enriched bacterial and fungal OTUs (bOTUs and fOTUs, respectively) were detected in mutants in comparison with their respective controls. For GLS, the highest numbers of differentially abundant bOTUs were observed in TU3 and cyp79B3 (Fig. 2A, Table S2). bOTUs representing the genera Azospirillum and Fluviicola showed the highest enrichment in several GLS mutants. Other significantly enriched genera included Nocardioides and Streptomyces in mutants such as TU3, myb51 and cyp79B3. In addition, both Massilia and Flavobacterium were significantly enriched in cyp79B3 and TU3. fOTUs assigned to the genera Sporobolomyces and Emericellopsis were the most significantly enriched cyp79B3, 
cyp79B2cyp79B3 and TU3 GLS mutants (Fig. 2A, Table S3). The genus Falciphora was enriched in both cyp79B3 and cyp79B2cyp79B3. fOTUs belonging to Metarhizium, Acremonium and Cyphellophora were enriched in cyp79B3, while Tetragoniomyces and an unidentified genus in the family Pyronemataceae were enriched in the double mutant cyp79B2 cyp79B3 (Fig. 2A, Table S3). In addition, the genera Clonostachys and Trichoderma were enriched in TU3 and pad2-1, respectively, while Fusarium was enriched in pen3-1-NahG.

In the FLV dataset, we found fewer differentially abundant bacterial genera (Fig. 2B, Table S4). bOTUs belonging to Flavobacterium were generally enriched in the FLV mutants, and Rhodanobacter and Azospirillum were significantly enriched in tt7-7 and pap1-D, respectively. For fungi, we observed enriched genera including Alternaria in tt5, Sporobolomycetes and Emericellopsis in pap1-D and Cladosporium and Psathyrella, and an unidentified member of the class Agaricomycetes in tt7-7 (Fig. 2B, Table S4). Both tt7-7 and tt5 had an increased abundance of Apiotrichum. In tt3, Apodus and an unidentified member of the class Leotiomycetes were enriched. Surprisingly, we did not detect any significant enrichment of fungi in the tt3-1tt5-1 double mutation.

Defense signaling mutants including dde2, etr1-3 and 35S::ERF were enriched in bOTUs assigned to Azospirillum, Herminiimonas, Fluviicola and Flavobacterium (Fig. 2C, Table S5). Similarly, FAD mutants fad3-2 and fad7-1fad8-1 were enriched in Azospirillum and Fluviicola. Comparing the ET mutants and their reference, etr1-3 had a higher number of enriched OTUs compared to 35S::ERF. Streptomycetes, Gaiella and Norcardioides were enriched in etr1-3 only. Noviherbaspirillum and Candidatus Udaeobacter were enriched in ABA mutants. The fungal class Sporobolomyces was significantly enriched in mutants dde2, fad3-2 and fad7-1fad8-1(Fig. 2C, Table S6). Similarly, Emericellopsis was highly enriched in dde2, etr1-3, and npr1-2. The ethylene mutants etr1-3 and 35S::ERF, had increased abundances of Saitozyma and an unidentified member of Pyronemataceae. The aba mutants showed an increased abundance of Alternaria and fad3-2 had an increased 
abundance of Tetragoniomyces, Solicoccozyma and Clonostachys, while fad7-1fad8-1 had a higher abundance of Mortierella, Tremellomycetes and Mortierellomycetes and an unidentified member of Eurotiomycetes (Fig. 2C, Table S6).

\section{GLS mutation affect microbial composition in roots}

Bacterial alpha diversity was not significantly different between GLS mutants and their controls, although the GLS mutants gsm1-1 and pad2-1 showed the lowest bacterial alpha diversities (Fig. 3A, Additional file 1: SF 5A, 6A). GLS affected fungal alpha diversity (observed, $\mathrm{P}<0.05$ ) (Fig. 3B, Additional file 1: SF 5B, 6B) but an ANOVA pairwise comparison showed no marked significant differences between mutants and control. A cyp79B2 mutation showed increased fungal alpha diversities but we noticed a decrease in the other mutants; cyp79B2cyp79B3, myb51 and pad3-1 had the lowest fungal alpha diversities when compared to Col-0.

Bacterial beta diversity analysis and visualization using PCoA ordination plots showed a clear separation of GLS mutants from Col-0 (Fig. 4A). We observed similar distinct clustering in the fungal PCoA plots, except that the Col-0 and pen3-1-NahG clustered together (Fig. 4A). PERMANOVA on the microbial communities revealed significant differences in the GLS dataset (Adonis, bacteria: $\mathrm{R}^{2}=$ 0.31, $\mathrm{P}<0.001$; fungi: $\mathrm{R}^{2}=0.27, \mathrm{P}<0.001$, Table 1) and thus corroborated the PCoA plots. To determine community distinctiveness as shown in PCoA plots for the GLS mutants and control, we performed PERMANOVA by comparing individual mutants to their wild type. Bacterial communities were strongly affected in cyp79B3 (Adonis, $\mathrm{R}^{2}=0.51, \mathrm{P}>0.01$ ) and TU3 (Adonis, $\mathrm{R}^{2}=$ $0.50, \mathrm{P}>0.01)$. A similar analysis revealed minor but significant effects $(\mathrm{p}<0.05)$ of genotypes on fungal communities. Unlike bacteria, the effects of TU3 and cyp73B3 on fungal communities were comparable $(\mathrm{p}<0.05)$ to the other GLS mutants. 
While we did not find significant differences in bacterial alpha diversities (Fig. 3A, Additional file

1: SF 5A, 6A) in the FLV dataset, fungal alpha diversity was significantly affected (observed, $\mathrm{P}<0.05$ )

(Fig. 3B, Additional file 1: SF 5B, 6B). However, an ANOVA pairwise comparison showed no marked differences in the alpha diversities between individual mutants and controls. PCoA ordination plots showed clustering of mutants from the controls in both bacterial and fungal datasets (Fig. 4B), but a PERMANOVA analysis only showed significant difference in the community structures of bacteria (Adonis, $\mathrm{R}^{2}=0.36, \mathrm{P}<0.001$, Table 1). Further data splitting and PERMANOVA analysis on individual mutants and their respective controls revealed that the tt7-7 mutation had the strongest

\section{Microbial composition in roots of defense signaling mutants}

Microbial diversity analysis showed that alpha diversities in both bacterial and fungal communities for the signaling mutants and their controls were non-significant (Figs. 3A, 3B; Additional file 1: SF5 and SF6). Both bacterial and fungal beta diversities revealed a clear separation of mutants and their controls on PCoA ordination plots (Figs. 4C). PERMANOVA analysis indicated a significant effect of defense signaling mutations on bacterial communities $\left(\mathrm{R}^{2}=0.39, \mathrm{P}<0.001\right.$ Table 1$)$ but not on fungal communities. Data partitioning for individual mutants and their respective controls showed minor but significant effects of most of the defense signaling mutants and controls on fungal communities (Table 1). 


\section{Discussion}

Advances in plant genetics have improved our understanding of functional plant genomics in the context of plant microbe-interactions. The assembly of host-specific microbiomes involves a direct or indirect effect of gene regulatory functions underlying biochemical or defense signaling processes in plants $(23,27,40)$. Characterizing microbiomes of mutants impaired in the synthesis of specific compounds could give us insights into host genotype-microbiome relations, enabling us in the end, to manipulate microbiomes for desired outcomes. In this study, we profiled the microbiomes of a wide range of Arabidopsis mutants and determined microbial relative abundance patterns and differentially abundant microbial taxa between mutants and their background controls.

\section{GLS have distinct effects on the host root-associated microbiome}

The identification of a range of bacterial and fungal taxa affected by the different GLS mutants demonstrated that GLS has distinct effects on specific microbial groups. Mutations in genes located at the initial steps of a biosynthetic pathway generally have more pronounced effects on the hostassociated microbiota (23). Similarly, we found that both cyp79B3 and TU3 that carry mutations upstream in the GLS pathway had the highest effect on bOTUs. Nonetheless, the mutants gsm1-1, cyp79B2 and the double mutant cyp79B2cyp79B3 (with total disruption in both the indolic GLS and camalexin pathways) $(41,42)$ with gene disruptions in the initial steps of the pathway similar to TU3 and cyp79B3 did not show the same dramatic effect on bOTUS. Moreover, the disruption of cyp79B2 and cyp79B3 genes occurring in the same step of the indolic GLS pathway or the TU1 and TU3 genes of the aliphatic GLS pathway, distinctively affected microbial composition in the respective mutants carrying these mutations. Both cyp79B2 and cyp79B3 mutants are disrupted in genes known for the same function (conversion of tryptophan to indole-3-acetaldoxime) and are therefore considered functionally redundant (41). However, Brader et al. (43) showed differences in the induction of the 
cyp79B2 and cyp79B3 genes upon treatment with culture filtrates of the bacterium Erwinia carotovora. Hence, it is possible that unknown enzymatic and pleiotropic activities of the cyp79B2 and cyp79B3 genes contributed to the observed differences of microbial structures. Furthermore, Ludwig-Müller et al. (44) reported that several TU mutants developed varying degrees of clubroot disease symptoms caused by Plasmodiophora brassicae. In that study, different contents of GLS intermediate products were found in the TU lines. Buxdorf et al. (45) showed that fungal pathogens, such as the Brassicaceae pathogen Alternaria brassicola, were less sensitive to GLS when compared to non-adapted fungi. Furthermore, B. cinerea isolates were reported to display strain-specific sensitivity to GLS (46). The increased abundance of individual bacterial genera such as Azospirillum and Fluviicola as well as fungal genera Sporobolomyces and Emericellopsis in several GLS mutants could further confirm the "gating" role of specific metabolic profiles on individual microbial taxa $(23,27)$. Plant metabolic compounds with antimicrobial properties including GLS are known to constitute the root rhizoplane's boundary layers that modulate host root microbiome assembly $(23,47,48)$. Altogether, these data confirm the GLS specific effects on microbial communities.

Genera such as Azospirillum, Nocardioides and the plant beneficial taxa Streptomyces and Flavobacterium were affected in some of the GLS mutants. Also, Azospirillum contains several beneficial species, widely known for their plant growth promoting traits including nitrogen fixation and the synthesis of phytohormones and other compounds required for both biotic and abiotic tolerance (49). In particular, the upstream mutations cyp79B3 and TU3 mutants were highly enriched in Flavobacterium and Massilia. The yeasts Sporobolomyces, which were enriched mostly in the FAD, dde2 and npr1-2 mutants, are abundant members of the plant mycobiome $(50,51)$ and have been shown to have antagonistic effects against pathogens (52). Some members of Emericellopsis are known to possess biocontrol traits via the antimicrobial compound emericellipsin A, which have been shown to suppress the pathogen Aspergillus niger (53). 
Furthermore, the clear separation of GLS mutants from Col-0 observed on a PCoA plot further supports our findings. In particular, the bacterial communities were strongly affected in cyp79B3 and TU3 mutants. Similarly, Micallef and Colón-Carmona (54) showed that overexpressed Arabidopsis mutants atr1D (with elevated GLS levels) and the double mutant cyp79B2cyp79B3 had distinct rhizobacterial community structures. Bressan et al. (21) also showed distinct differences in microbial community composition of the GLS mutant cyp79A1 and the control Col-0. Together, these findings confirm our hypothesis of the effect of gene mutations in metabolic pathways on the innate plant recruitment potential.

\section{Flavonoid affect the host root-associated microbiomes}

By comparing the microbiomes of FLV mutants and controls, we observed distinct effects on microbial composition. In addition, significant differences between mutants and the controls were observed at higher taxonomic ranks including Actinobacteria, Thermoleophilia and Verrucomicrobiae as well as the fungal classes Sordariomycetes and Eurotiomycetes. Previously, it has been shown that FLVs affects microbial community structures (55) while other studies have reported non-significant effects of some FLV mutations on microbial community structures (20). Surprisingly, while mutations in single genes (in tt3 and tt5) significantly affected both bacterial and fungal communities, the double mutant $\mathrm{tt} 3-1 \mathrm{tt} 5-1$ only had significant effects on fungal communities. Associated pleiotropic effects of the mutations resulting in unpredictable FLV profiles $(56,57)$ could explain this observation.

Arabidopsis predominantly produce the flavonols, kaempferol and quercetin glycosides (58). The observed effect of the tt7-7 mutation in the present study suggests a more pronounced effect of the depletion of both quercetin and kaempferol on bacterial communities. Gene disruptions in the tt mutants have been shown to accumulate varying FLV profiles $(14,8,59,60)$. For example, the $t \mathrm{t} 3$ 
mutant with disruption in the dihydroflavonol 4-reductase gene located downstream in the FLV biosynthetic pathway was shown to accumulate appreciate levels of the intermediate products quercetin and kaempferol (14). The tt5 with a disruption in the chalcone-flavanone isomerase gene was reported to lack flavonols but had detectable levels of the intermediates naringenin chalcone, chalcone and flavanone (14). Kaempferol and naringenin inhibits spore germination of plant pathogens (61) while quercetin enhances mycorrhizal-plant symbiosis by stimulating host penetration and hyphal growth (7). The biocontrol activity of the isoflavonoid flavans and flavanones (16) extend to their ability to modulate genes regulating the synthesis of the antifungal compound 2,4diacetylphloroglucinol and pyoluteorin by the biocontrol agent Pseudomonas fluorescens CHA0 (62). In addition, Vandeputte et al. $(61,62)$ demonstrated naringenin and catechin produced in higher plants to be important in reducing the production of quorum sensing-controlled virulence factors in Pseudomonas aeruginosa PAO1. The fact that we only observed few differentially abundant bacterial taxa when compared to fungi suggests a stronger effect of FLVs on fungi. The observed differential effect of FLVs on fungal taxa corroborates the finding of distinct effects of FLVs on individual fungi such as arbuscular mycorrhizal fungi (63). The strong enrichment of the plant pathogenic genus Alternaria in tt5 mutants indicates that naringenin could play a defensive role against plant pathogens, as suggested previously (62). In summary, like the GLS, FLVs could have a strong innate microbial recruitment role and are thus important for the modulation of root-associated microbiomes.

\section{Defense signaling mutations affect host root-microbiome assembly.}

Similar to the GLS and FLV mutations, DSMs strongly affected microbial communities with specific effects of individual mutants on both bacterial and fungal communities. The observed differential effects of both ET insensitive and overexpressed mutants on microbial communities confirm previous studies $(64,65)$. The detection of ABA mutation affecting microbial taxa, for example, the enrichment of Acidobacteria, Alphaproteobacteria and Sordariomycetes taxa in aba mutant compared to Ler-0 
support that ABA preferentially affect Arabidopsis-root associated microbiota (66). Studies have shown that $\mathrm{ABA}$ is a key regulator of defense and in many instances was found to suppress the activation of plant defense genes $(67,68,69)$. While the mechanism of negative defense modulation activity of $\mathrm{ABA}$ is not well understood, we speculate that $\mathrm{ABA}$ depletion of core microbial taxa in Arabidopsis, Alphaproteobacteria and Sordariomycetes $(70,71)$, detected in Ler- 0 in this study could contribute to the weak defense against pathogens.

FAD is pivotal in the hormonal signaling network by modulating both the SA (72) and JA pathways (73), and its role in mediating plant-microbe interactions has been reported $(19,28)$. Our study revealed distinct differential effect of FAD mutants on microbial community structures but both mutants revealed increased enrichment of the bacterial genera Azospirillum and fungal Sporobolomyces (for fad7-1fad8-1) or unidentified genus in the Sporidiobolaceae (for fad3-2). This finding corroborates a previous study where the Arabidopsis triple mutant fad 3 fad 7 fad 8 had higher bacterial diversity compared to the control, with several species within Alpha- and Gammaproteobacteria enriched in the FAD mutant (28). Moreover, differential effects of FAD genes on specific microbial members have been reported. For instance, while the FAD3 gene was shown to be unresponsive upon inoculation of the bacterial pathogen Xanthomonas campestris (72), FAD7 genes are known to be induced by fungal effectors (74). The observation of differential effects of FAD single or double mutations on microbial communities indicate specific effects of individual FAD genes on the host associated microbiomes. However, hormonal crosstalk mechanisms could be involved in the observed effects of FAD mutation on microbial communities.

In addition, the observed strongest effect of $\mathrm{ABA}$ and the FAD double mutant on bacterial communities (PERMANOVA, 39\% and 46\%) could further suggest a higher pronounced modulating role of these molecules on bacterial community assembly. The JA mutant dde 2 significantly affected both bacterial and fungal communities. This finding corroborates a study in which JA mutants myc2 
and med25 were reported to sculpt distinct bacterial and archaeal compositions when compared to the control (64). In general, we have demonstrated that mutations in signaling pathways affect microbial community structures, while differentially affected microbial taxa further supports this. These results are consistent with previous findings (27), however, the inclusion of a range of mutants in different signaling pathways in the present study has expanded on the scope of the subject.

A cascading effect of immune system activation is the induction of systemic acquired resistance (SAR) and induced systemic resistance (ISR), with the phytohormones SA, JA, ABA and ET acting as DSMs (9). Hormonal cross-talk mechanisms implicate the interaction of these signaling hormones in fine-tuning defense against biotic and abiotic stresses $(9,75)$. For example, ABA is reported to be involved in SA-JA-ET networks, and therefore important in subsequent defense activations $(9,76-$ 78). This interconnectedness in signaling pathways presents a high complexity, thus, mutant lines with several mutations with the possible elimination of overlapping defense-signaling functions are highly recommended (27). Pleiotropic effects arising, for instance, from a disruption in the NPR1 gene makes it impossible to examine the exact effect of SA on microbial community structures. As such, it is difficult to make definitive conclusions with the specific mutations on microbial communities. We therefore suggest that a detailed analysis could include mutants with complete abolishment of interactive pathways, complemented with other omics analysis techniques.

\section{Conclusions}

Plant metabolic and defense signaling pathways are an integral part of the innate sculpting mechanisms that regulate the assembly of host-associated microbiota. By using different Arabidopsis mutants and their background controls, we showed that both secondary metabolism and DSMs affect bacterial and fungal community structures. We found the bacterial taxa Actinobacteria, Thermoleophilia and Verrucomicrobiae and the fungal taxa Eurotiomycetes, Olpidiomycetes and 
Sordariomycetes to be the most highly affected between mutants and their controls. Differential analysis at lower taxonomic levels revealed significantly affected genera between Arabidopsis GLS, FLVs and defense signaling mutants and their respective controls. These results strongly support the perception that many plant secondary metabolites and DSMs affect microbiome assembly. Our findings suggest that disruption of secondary metabolite or signaling pathways affect the innate mechanisms that modulate root-associated microbiome assembly. Further analysis complemented by other omics techniques could provide an indepth understanding of the plant-omics links, thus facilitating the utilization of microbiome solutions for sustainable plant production.

\section{Acknowledgements}

We thank Profs Judith Bender (Brown University, USA), Wendy Peer (University of Maryland, USA) and Paul E. Staswich (University of Nebraska, USA) for kindly providing the Arabidopsis mutants. We thank Simone Ena Rasmussen and Mathilde Schiøtt Dige for their excellent laboratory technical assistance.

\section{Funding}

This work was financially supported by Aarhus University (project number 22550) and the Independent Research Fund Denmark (DFF), grant number 6111-00065B.

\section{Availability of data and materials}

The MiSeq paired end reads for bacterial 16 s rRNA gene (V3-V4) and fungal ITS2 regions have been deposited in the NCBI Sequence Read Archive (SRA) database under the accession number PRJNA579829. 
ENK, RS and MN conceived the study, participated in its design, and wrote the paper. ENK and RS conducted the experiment and analyzed the data. All authors read and approved the manuscript.

420 Ethics approval and consent to participate

421 Not applicable.

422 Competing interests

423 The authors declare that they have no conflict of interest.

\section{List of Tables}

Table 1. Permutation analysis of variance (PERMANOVA) for individual mutants and background controls. Adonis tests were based on weighted UniFrac matrices for bacterial and Bray-Curtis distance matrices for fungal community dissimilarity assessment using 1000 permutations.

\begin{tabular}{|c|c|c|c|}
\hline Dataset/ Factor & Genotype description & Bacteria $\left(\mathbf{R}^{2}\right)$ & Fungi $\left(R^{2}\right)$ \\
\hline GLS (Mut1) & GLS mutants and wild type & $0.31 * * *$ & $0.27 * * *$ \\
\hline FLV (Mut2) & FLV mutants and wild types & $0.36 * * *$ & - \\
\hline DSM (Mut3) & $\begin{array}{l}\text { Defense signaling mutants } \\
\text { and wild types }\end{array}$ & $0.39 * * *$ & -- \\
\hline \multicolumn{4}{|l|}{ GLS } \\
\hline Col-0_cyp79B3 & IGS partial disruption) & $0.51 * *$ & -- \\
\hline $\begin{array}{l}\text { Col- } \\
\text { 0_cyp79B2cyp79B3 }\end{array}$ & $\begin{array}{l}\text { Lacks IGS and camalexin } \\
\text { (Blocked in the production } \\
\text { of } \mathrm{I} 3 \mathrm{AOx}) \text { ) }\end{array}$ & $0.43^{*}$ & $0.31 *$ \\
\hline Col-0_myb51 & IGS synthesis disruption & $0.36^{*}$ & $0.24 *$ \\
\hline Col-0_gsm1-1 & $\begin{array}{l}\text { Reduced amounts of many } \\
\text { aliphatic glucosinolates }\end{array}$ & $0.33 *$ & $0.30 *$ \\
\hline
\end{tabular}




\begin{tabular}{|c|c|c|c|}
\hline Col-0_TU3 & $\begin{array}{l}\text { Deficient in aliphatic GLS } \\
\text { with heptyl and octyl core } \\
\text { groups }\end{array}$ & $0.50 * *$ & $0.37 *$ \\
\hline Col-0_pad2-1 & Partially blocks camalexin & -- & $0.38^{*}$ \\
\hline Col-0_pen3-1-NahG & $\begin{array}{l}\text { Disruption in both IGS } \\
\text { synthesis and SA signaling } \\
\text { pathways. }\end{array}$ & $0.27 *$ & -- \\
\hline \multicolumn{4}{|l|}{ FLV } \\
\hline Ler-0_tt7-7 & $\begin{array}{l}\text { Deficient in flavonoid 3'- } \\
\text { hydroxylase activity and } \\
\text { lacks orthodihydroxy } \\
\text { flavonoids such as quercetin } \\
\text { and kaempferol }\end{array}$ & $0.41 * *$ & $0.22 *$ \\
\hline Ler-0_tt3 & $\begin{array}{l}\text { Excess quercetin, } \\
\text { kaempferol }\end{array}$ & $0.22 *$ & -- \\
\hline Ler-0_tt5 & $\begin{array}{l}\text { Low-level quercetin } \\
\text { production }\end{array}$ & $0.30 *$ & $0.25^{*}$ \\
\hline Ler-0_tt3-1tt5-1 & $\begin{array}{l}\text { Double mutation, disruption } \\
\text { of the synthesis of brown } \\
\text { pigment }\end{array}$ & -- & $0.16^{*}$ \\
\hline Col-0_pap1-D & $\begin{array}{l}\text { Overexpressed } \\
\text { (anthocyanin) mutant }\end{array}$ & $0.37 *$ & $0.30 *$ \\
\hline \multicolumn{4}{|l|}{ DSMs } \\
\hline Col-0_dde2 & JA deficient & $0.33 *$ & $0.34 *$ \\
\hline Ler-0_aba3-2 & ABA deficient & $0.39 * *$ & $0.30 *$ \\
\hline Col-0_etr1-3 & Ethylene responsive & $0.47 *$ & $0.31 *$ \\
\hline Col-0_35S::ERF & Ethylene (overexpressed) & $0.32 *$ & $0.24 *$ \\
\hline Col-0_fad3-2 & $\begin{array}{l}\text { Fatty acid desaturase (FAD) } \\
\text { deficient }\end{array}$ & -- & $0.18^{*}$ \\
\hline Col-0_fad7-1fad8-1 & FAD double mutation & $0.46 * *$ & -- \\
\hline
\end{tabular}

429 Significance of test indicated as $* * *$ for $\mathrm{p}<0.001, * * \mathrm{p}>0.01, * \mathrm{p}<0.05$ and $\mathrm{R}^{2}$ for the proportion of 430 variation explained. I3AOx (Indole-3-aldoxime) IGS (indole glucosinolate) 


\section{List of figures}

Figure 1. Bar plots showing mean distribution of reads assigned at class level in A) GLS mutants and their controls, B) FLV mutants and their controls and C) defense signaling mutants and their controls. Mutants were compared to their background controls by ANOVA, followed by the Tukey-Kramer post hoc test $(\mathrm{p}<0.05)$ using the Benjamini and Hochberg $(\mathrm{BH})$ FDR for multiple comparisons. Only top 10 taxa were used and taxa with small effect sizes were removed by filtering (effect size $=0.8$ ). The black and white bar plots represent controls and mutants respectively. Error bars represent standard deviations. The analysis was performed using the STAMP software (v2.1.3)

Figure 2: Network showing microbial differential analysis between mutants and controls. Bacterial and fungal genera that were significantly affected are shown for A) GLS mutants and control. B) FLV mutants and control C) defense signaling mutants and control. Analysis performed using DESeq2. Enrichment in mutants are shown in red and in blue for controls. Line width indicates the significant level of effect.

Figure 3. Alpha diversity estimated using Shannon diversity for the respective genotypes.

A) Bacterial and (B) fungal Shannon diversity in different genotypes. Samples with at least 3 replicates for both bacteria and fungi (with bacterial and fungal reads >800 and >500 respectively) are shown.

Figure 4. Principal coordinate analysis (PCoA) of weighted UniFrac distances (bacterial) and BrayCurtis (fungal) between GLS (A), FLV (B) and DSM (C) genotypes. Individual genotypes and sample groups are shown in different colours and shapes. 


\section{Additional file 1}

456

457

458

\section{Supplementary figures}

Figure S1. Arabidopsis growth and harvesting scheme. A) Arabidopsis growing under greenhouse conditions. B) Summer conditions (temperature and relative humidity (RH) for the growth of Arabidopsis in greenhouses. Each bar represent daily readings (at hourly intervals) Weather data was obtained from the DMI Dalmose station. C) Arabidopsis with the roots (circled in red), the part harvested for analysis D) Root samples from individual plants in same pots were pooled into in a collection tube (representing a replicate) and subsequently frozen.

Figure S2. Schematics of biosynthetic and signaling pathways. A) Biosynthetic pathway of aliphatic and indolic GLS in Arabidopsis. Schematic overview of genes involved at the different steps of the pathway. Disrupted genes from which mutants were derived are in bold while mutants are shown in blue. Adapted from suppl. ref: 1, 2, 3, 4 and 5. IAN (Indole-3-acetonitrile), TSB1 (tryptophan synthase beta subunit 1). B) The FLV biosynthetic pathway. The transparent testa (tt) mutations in mutants (parentheses) used in this study are indicated. Enzyme abbreviations: CHI, chalcone isomerase; CHS, chalcone synthase; DFR, dihydroflavonol reductase; F3H, flavonol 3-hydroxylase; FLS, flavonol synthase. *(double mutant). Adapted from suppl ref. 6. C) The schematics of signaling pathways. The phytohormones salicylic acid (SA), jasmonic acid (JA), ethylene (ET), abscisic acid (ABA) mediate defense signaling in plants. Fatty acid desaturase (FAD) is also involved in defense including the regulation of JA and SA pathways. Genes of interest (bold) in a pathway and mutants (blue) carrying a mutation in these genes are represented. Defense cross-talk interactions that finetune defense signaling outcomes is also shown. NPR1 (Non-expressor of PR genes1), AAO3 (Abscisic acid synthase), AOS (Allene oxide synthase). 
Figure S3. Box plots showing the distribution of the number of reads and OTU distribution in each sample used in this study. A) Bacterial reads and B) OTU distribution, C) fungal reads and D) OTU distribution.

Figure S4. Class-level relative abundances of microbial communities Arabidopsis genotypes. a) Bacterial relative abundance at class level in the different GLS mutants and controls, Col-0. b). Bacterial relative abundance at class level in the different GLS mutants and controls, Col-0. c). Bacterial relative abundance at class level in the different plant FLV mutants and controls, Col-0 and Ler-0. d) Fungal relative abundance at class level in the different FLV mutants and controls, Col-0 and Ler-0. e) Bacterial relative abundance at class level in the different defense signaling mutants and controls, Col-0 and Ler-0. f) Fungal relative abundance at class level in the different defense signaling mutants and controls, Col-0 and Ler-0.

Figure S5. Observed bacterial (A) and fungal (B) OTU richness in the different genotypes. Samples with at least three replicates (with bacterial and fungal reads $>800$ and $>500$ respectively) are shown.

Figure S6. Alpha diversities estimated in the respective genotypes using observed OTU richness and Shannon diversity matrices for A) Bacteria and B) Fungi. All sample with reads $>800$ for bacterial and $>500$ fungal are presented.

Table S1. List of Arabidopsis genotypes used for the study. 
Table S1. Differentially abundant bacterial OTUs on roots of Col-0 and GLS mutants as determined using DESeq2.

Table S2. Differentially abundant fungal OTUs on roots of Col-0 and GLS mutants; as determined using DESeq2.

Table S3. Differentially abundant bacterial OTUs on roots of Ler-0 or Col-0 and FLV mutants as determined using DESeq2.

Table S4. Differentially abundant fungal OTUs on roots of Ler-0 or Col-0 and FLV mutants as determined using DESeq2.

Table S5. Differentially abundant bacterial OTUs on roots of Col-0 or Ler-0 and signaling mutants as determined using DESeq2.

Table S6. Differentially abundant fungal OTUs on roots of Col-0 or Ler-0 and signaling mutants as determined using DESeq2.

\section{References}

1. Chisholm ST, Coaker G, Day B, Staskawicz BJ. Host-microbe interactions: shaping the evolution of the plant immune response. Cell. 2006;124(4):803-14.

2. Jones JDG, Dangl L. The plant immune system. Nature. 2006; 444:323-9.

3. Kliebenstein DJ. Secondary metabolites and plant/environment interactions: A view through Arabidopsis thaliana tinged glasses. Plant, Cell Environ. 2004;27(6):675-84.

4. Kirkegaard JA, Sarwar M. Biofumigation potential of brassicas I. Variation in glucosinolate 
profiles of diverse field-grown brassicas. 1998;71-89.

520 5. Tierens KF, Thomma BP, Brouwer M, Schmidt J, Kistner K, Porzel a, et al. Study of the role 521 of antimicrobial glucosinolate-derived isothiocyanates in resistance of Arabidopsis to microbial pathogens. Plant Physiol. 2001;125:1688-99.

6. Angus JF, Gardner PA, Kirkegaard JA, Desmarchelier JM. Biofumigation: Isothiocyanates released from brassica roots inhibit growth of the take-all fungus. Plant Soil. 1994;162:107-12.

7. Cesco S, Mimmo T, Tonon G, Tomasi N, Pinton R, Terzano R, et al. Plant-borne flavonoids released into the rhizosphere: Impact on soil bio-activities related to plant nutrition. A review. Biol Fertil Soils. 2012;48(2):123-49.

8. Buer CS, Imin N, Djordjevic MA. Flavonoids: New roles for old molecules. J Integr Plant Biol. 2010;52: 98-111.

9. Pieterse CMJ, Leon-Reyes A, Van Der Ent S, Van Wees SCM. Networking by small-molecule hormones in plant immunity. Nat Chem Biol. 2009;5(5):308-16.

10. Hacquard S, Spaepen S, Garrido-Oter R, Schulze-Lefert P. Interplay between innate immunity and the plant microbiota. Annu Rev Phytopathol. 2017;55(1):565-89.

53411 Graser G, Oldham NJ, Brown PD, Temp U, Gershenzon J. The biosynthesis of benzoic acid glucosinolate esters in Arabidopsis thaliana. Phytochemistry. 2001;57(1):23-32.

12. Halkier BA, Gershenzon J. Biology and biochemistry of glucosinolates. Annu Rev Plant Biol. 2006;57:303-33.

13. Haughn GW, Davin L, Giblin M, Underhill EW. Biochemical genetics of plant secondary metabolites in Arabidopsis thaliana. Plant Physiol.1991;217-26. 
14. Peer WA, Brown DE, Tague BW, Muday GK, Taiz L, Murphy AS. Flavonoid accumulation patterns of transparent testa mutants of Arabidopsis. Plant Physiol. 2001;126(2):536-48.

15. Saito K, Yonekura-Sakakibara K, Nakabayashi R, Higashi Y, Yamazaki M, Tohge T, et al. The flavonoid biosynthetic pathway in Arabidopsis: Structural and genetic diversity. Plant Physiol Biochem. 2013;72:21-34.

16. Weston LA, Mathesius U. Flavonoids: their structure, biosynthesis and role in the rhizosphere, including allelopathy. J Chem Ecol. 2013;39(2):283-97.

17. Staswick PE, Yuen GY, Lehman CC. Jasmonate signaling mutants of Arabidopsis are susceptible to the soil fungus Pythium irregulare. Plant J. 1998;15(6):747-54.

18. Soliman S, El-Keblawy A, Mosa KA, Helmy M, Wani SH. Understanding the phytohormones biosynthetic pathways for developing engineered environmental stress-tolerant crops. Biotechnologies of crop improvement, Volume 2: Transgenic Approaches. 2018: 1-485.

19. Kachroo P, Shanklin J, Shah J, Whittle EJ, Klessig DF. A fatty acid desaturase modulates the activation of defense signaling pathways in plants. Proc Natl Acad Sci. 2002;98(16):9448-53.

20. Badri D V., Quintana N, El Kassis EG, Kim HK, Choi YH, Sugiyama A, et al. An ABC transporter mutation alters root exudation of phytochemicals that provoke an overhaul of natural soil microbiota. Plant Physiol. 2009;151(4):2006-17.

21. Bressan M, Achouak W, Berge O. Exogenous glucosinolate produced by transgenic Arabidopsis thaliana has an impact on microbes in the rhizosphere and plant roots. ISME J. 2009;3:1243-57.

22. Hu L, Robert CAM, Cadot S, Zhang X, Ye M, Li B, et al. Root exudate metabolites drive 
plant-soil feedbacks on growth and defense by shaping the rhizosphere microbiota. Nat Commun. 2018;9(1):1-13.

23. Kudjordjie EN, Sapkota R, Steffensen SK, Fomsgaard IS, Nicolaisen M. Maize synthesized benzoxazinoids affect the host associated microbiome. Microbiome. 2019;1-17.

24. Stringlis IA, Yu K, Feussner K, de Jonge R, Van Bentum S, Van Verk MC, et al. MYB72dependent coumarin exudation shapes root microbiome assembly to promote plant health. Proc Natl Acad Sci. 2018; 115(22): 5213-5222.

25. Lundberg DS, Teixeira PJPL. Root-exuded coumarin shapes the root microbiome. Proc Natl Acad Sci. 2018;115 (22): 5629-5631.

26. Voges MJEEE, Bai Y, Schulze-Lefert P, Sattely ES. Plant-derived coumarins shape the composition of an Arabidopsis synthetic root microbiome. Proc Natl Acad Sci. 2019;116(25):12558-12565.

27. Lebeis SL, Paredes SH, Lundberg DS, Breakfield N, Gehring J, McDonald M, et al. Salicylic acid modulates colonization of the root microbiome by specific bacterial taxa. Science. 2015;349(6250):860-4.

28. Kniskern JM, Traw MB, Bergelson J. Salicylic acid and jasmonic acid signaling defense pathways reduce natural bacterial diversity on Arabidopsis thaliana. Mol Plant Microbe Interact. 2007;20(12):1512-22.

29. Shirley BW, Kubasek WL, Storz G, Bruggemann E, Koornneef M, Ausubel FM, et al. Analysis of Arabidopsis mutants deficient in flavonoid biosynthesis. Vol. 8, The Plant journal. 1995. $659-71$. 
30. Ihrmark K, Bödeker ITM, Cruz-Martinez K, Friberg H, Kubartova A, Schenck J, et al. New primers to amplify the fungal ITS2 region - evaluation by 454-sequencing of artificial and natural communities. FEMS Microbiol Ecol . 2012;82: 666-677.

31. Klindworth A, Pruesse E, Schweer T, Peplies J, Quast C, Horn M, et al. Evaluation of general 16S ribosomal RNA gene PCR primers for classical and next-generation sequencing-based diversity studies. Nucleic Acids Res. 2013;41(1):e1.

32. Rognes T, Flouri T, Nichols B, Quince C, Mahé F. VSEARCH: a versatile open source tool for metagenomics. PeerJ. 2016;4:e2584.

33. Caporaso JG, Kuczynski J, Stombaugh J, Bittinger K, Bushman FD, Costello EK, et al. QIIME allows analysis of high-throughput community sequencing data. Nat Methods. 2010;7(5):3356.

34. R Core Team. R : A language and environment for statistical computing. 2017:(2).

35. Oksanen AJ, Blanchet FG, Friendly M, Kindt R, Legendre P, Mcglinn D, et al. Vegan: Community ecology package. 2019. Version 2.5-6.

36. McMurdie PJ, Holmes S, Kindt R, Legendre P, O'Hara R. phyloseq: An R package for reproducible interactive analysis and graphics of microbiome census data. PLoS One. 2013;8(4):e61217.

37. Parks DH, Tyson GW, Hugenholtz P, Beiko RG. STAMP: statistical analysis of taxonomic and functional profiles. Bioinformatics. 2014;30:3123-3124.

38. Parks DH and Beiko RG. Identifying biologically relevant differences between metagenomic communities. Bioinformatics. 2010;26:715-721. 
603

604

605

606

607

608

609

610

611

612

613

614

615

616

617

618

619

620

621

622

623

39. Love MI, Huber W, Anders S. Moderated estimation of fold change and dispersion for RNAseq data with DESeq2. Genome Biol. 2014;15(12):1-21.

40. Zhalnina K, Louie KB, Hao Z, Mansoori N, Da Rocha UN, Shi S, et al. Dynamic root exudate chemistry and microbial substrate preferences drive patterns in rhizosphere microbial community assembly. Nat Microbiol. 2018;3(4):470-80.

41. Zhao Y, Hull AK, Ecker JR, Normanly J, Chory J, Celenza JL. Trp-dependent auxin biosynthesis in. Genes Dev. 2002;3:100-12.

42. Glawischnig E. Camalexin. Phytochemistry. 2007;68(4):401-6.

43. Brader G, Tas E, Palva ET. Jasmonate-dependent induction of indole glucosinolates in Arabidopsis by culture filtrates of the nonspecific pathogen Erwinia carotovora. Plant Physiol. 2001;126(2):849-60.

44. Ludwig-Müller J, Pieper K, Ruppel M, Cohen JD, Epstein E, Kiddle G, et al. Indole glucosinolate and auxin biosynthesis in Arabidopsis thaliana (L.) Heynh. glucosinolate mutants and the development of clubroot disease. Planta. 1999;208(3):409-19.

45. Buxdorf K, Yaffe H, Barda O, Levy M. The effects of glucosinolates and their breakdown products on necrotrophic fungi. PLoS One. 2013;8(8).

46. Kliebenstein DJ, Rowe HC, Denby KJ. Secondary metabolites influence Arabidopsis/Botrytis interactions: Variation in host production and pathogen sensitivity. Plant J. 2005;44(1):25-36.

47. Reinhold-Hurek B, Bünger W, Burbano CS, Sabale M, Hurek T. Roots shaping sheir microbiome: Global hotspots for microbial activity. Annu Rev Phytopathol. 2015;53(1):40324. 
48. van der Heijden MGA, Schlaeppi K. Root surface as a frontier for plant microbiome research: Fig. 1. Proc Natl Acad Sci. 2015;112(8):2299-300.

49. Fukami J, Cerezini P, Hungria M. Azospirillum: benefits that go far beyond biological nitrogen fixation. AMB Express. 2018;8(1):1-12.

50. Sapkota R, Jørgensen LN, Nicolaisen M. Spatiotemporal variation and networks in the mycobiome of the wheat canopy. Front Plant Sci. 2017;8:1357.

51. Sapkota R, Knorr K, Jørgensen LN, Hanlon K a O, Nicolaisen M. Host genotype is an important determinant of the cereal phyllosphere mycobiome. New Phytol. 2015;207(4):113444.

52. Wachowska U, Borowska J. Antagonistic yeasts competes for iron with winter wheat stem base pathogens. Gesunde Pflanz. 2014;66(4):141-8.

53. Rogozhin EA, Sadykova VS, Baranova AA, Vasilchenko AS, Lushpa VA, Mineev KS, et al. A novel lipopeptaibol emericellipsin A with antimicrobial and antitumor activity produced by the extremophilic fungus emericellopsis alkalina. Molecules. 2018;23(11).

54. Micallef S, Colón-Carmona A. Genetic and developmental control of rhizosphere bacterial communities. Mol Microb Ecol Rhizosph. 2013;1:257-63.

55. Czaban W, Rasmussen J, Laursen BB, Vidkjær NH, Sapkota R, Nicolaisen M, et al. Multiple effects of secondary metabolites on amino acid cycling in white clover rhizosphere. Soil Biol Biochem. 2018;123.

56. Sugiyama A, Yazaki K. Flavonoids in plant rhizospheres: Secretion, fate and their effects on biological communication. Plant Biotechnol. 2014;31(5):431-43. 
645 57. Saslowsky DE, Dana CD, Winkel-shirley B. An allelic series for the chalcone synthase locus 646 in Arabidopsis. Gene. 2000;255:127-38.

647 648 649 650 651 652 653 654 655 656 657 658 659 660 661 662 663 664 665 666

58. Buer CS, Djordjevic MA. Architectural phenotypes in the transparent testa mutants of Arabidopsis thaliana. J Exp Bot . 2009;60: 751-763.

59. Padmavati M, Sakthivel N, Thara K V., Reddy AR. Differential sensitivity of rice pathogens to growth inhibition by flavonoids. Phytochemistry. 1997;499-502.

60. Werra P De, Tabacchi R, Keel C, Maurhofer M. Plant- and microbe-derived compounds affect the expression of genes encoding antifungal compounds in a Pseudomonad with biocontrol activity. Appl enviromental Microbiol. 2011;77(8):2807-12.

61. Vandeputte OM, Kiendrebeogo M, Rajaonson S, Diallo B, Mol A, Jaziri M El, et al. Identification of catechin as one of the flavonoids from combretum albiflorum bark extract that reduces the production of quorum-sensing-controlled virulence factors in Pseudomonas aeruginosa PAQ1. Appl Environ Microbiol. 2010;76(1):243-53.

62. Vandeputte OM, Kiendrebeogo M, Rasamiravaka T, Stévigny C, Duez P, Rajaonson S, et al. The flavanone naringenin reduces the production of quorum sensing-controlled virulence factors in Pseudomonas aeruginosa PAO1. Microbiology. 2011;157(7):2120-32.

63. Scervino JM, Ponce MA, Erra-Bassells R, Vierheilig H, Ocampo JA, Godeas A. Flavonoids exhibit fungal species and genus specific effects on the presymbiotic growth of Gigaspora and Glomus. Mycol Res. 2005;109(7):789-94.

64. Bodenhausen N, Bortfeld-Miller M, Ackermann M, Vorholt JA (2017) A synthetic community approach reveals plant genotypes affecting the phyllosphere microbiota. PLoS Genet (2017);10(4): e1004283. 
65 Doornbos RF, Geraats BPJ, Kuramae EE, Van Loon LC, Bakker P AHM. Effects of jasmonic acid, ethylene, and salicylic acid signaling on the rhizosphere bacterial community of Arabidopsis thaliana. Mol Plant Microbe Interact. 2011;24:395-407.

66. Carvalhais LC, Dennis PG, Badri D V, Kidd BN, Vivanco JM, Schenk PM. Linking jasmonic acid signaling, root exudates, and rhizosphere microbiomes. Mol Plant Microbe Interact. 2015;28(9):1049-58.

67. Adie BA, Pérez-Pérez J, Pérez-Pérez MM, Godoy M, Sánchez-Serrano JJ, Schmelz EA, et al. $\mathrm{ABA}$ is an essential signal for plant resistance to pathogens affecting JA biosynthesis and the activation of defenses in Arabidopsis. Plant Cell. 2007;19(5):1665-81. 68 Xiong L, Yang Y. Disease resistance and abiotic stress tolerance in rice are inversely modulated by an abscisic acid-inducible mitogen-activated protein kinase. Plant Cell. 2003;15:745-59.

69 Gao S, Guo W, Feng W, Liu L, Song X, Chen J, et al. LTP3 contributes to disease susceptibility in Arabidopsis by enhancing abscisic acid (ABA) biosynthesis. Mol Plant Pathol. 2016;17:412-26.

70 Hacquard Stephane, Garrido-Oter R, Anthonio G, Spaepen S, Ackermann G, Lebeis S, et al. Microbiota and host nutrition across plant and animal kingdoms. 2015;603-16.

71 Almario J, Jeena G, Wunder J, Langen G, Zuccaro A, Coupland G, Bucher M. Root-associated fungal microbiota of nonmycorrhizal Arabis alpina and its contribution to plant phosphorus nutrition. Proc Natl Acad Sci. 2017;114:9403-12.

72. Buell CR, Somerville SC. Expression of defense-related and putative signaling genes during tolerant and susceptible interactions of Arabidopsis with Xanthomonas campestris pv. 
691 73. Kirsch C, Takamiya-Wik M, Reinold S, Hahlbrock K, Somssich IE. Rapid, transient, and highly localized induction of plastidial $\omega-3$ fatty acid desaturase mRNA at fungal infection sites in Petroselinum crispum. Proc Natl Acad Sci. 1997;94(5):2079-84.

74. Avila CA, Arevalo-Soliz LM, Jia L, Navarre DA, Chen Z, Howe GA, et al. Loss of function 695 of fatty acid desaturase7 in tomato enhances basal aphid resistance in a salicylate-dependent manner. Plant Physiol. 2012;158(4):2028-41.

75. Pieterse CMJ, Van der Does D, Zamioudis C, Leon-Reyes A, Van Wees SCM. Hormonal 698 modulation of plant immunity. Annu Rev Cell Dev Biol. 2012;28(1):489-521.

699 76. Anderson JP. Antagonistic interaction between abscisic acid and jasmonate-ethylene signaling 700 pathways modulates defense gene expression and disease resistance in Arabidopsis. Plant Cell.

77. Flors V, Ton J, Van Doorn R, Jakab G, García-Agustín P, Mauch-Mani B. Interplay between JA, SA and ABA signalling during basal and induced resistance against Pseudomonas syringae and Alternaria brassicicola. Plant J. 2008;54(1):81-92. 


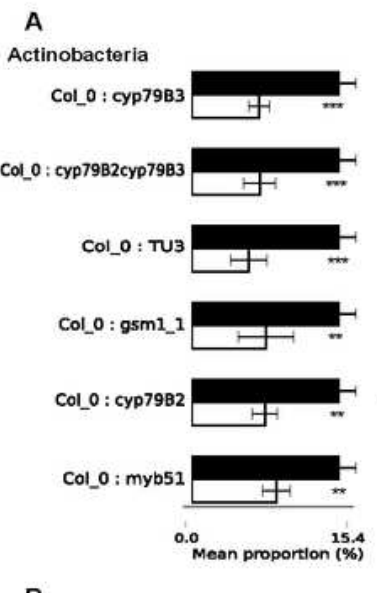

B

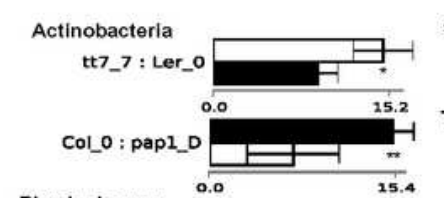

Phycisphaerae

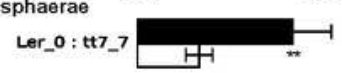

Ler_o : tt5
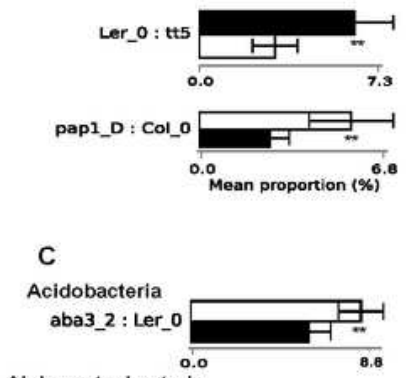

Alphaproteobacteria

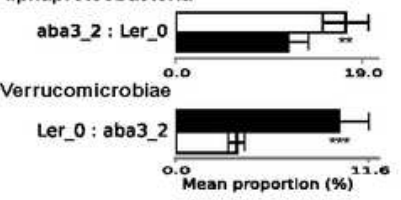

Bacteria

Thermoleophilia

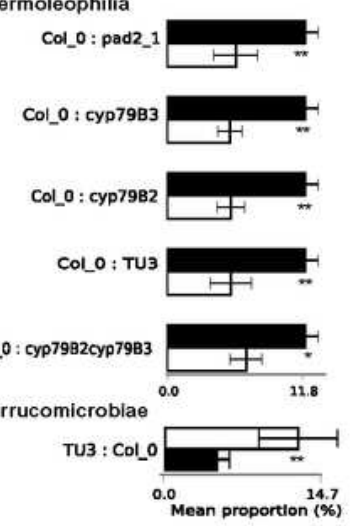

subgroup_6

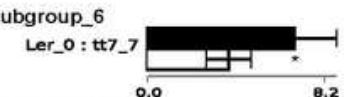

Thermoleophilia $0.0 \quad 0.2$

Col_o : pap1_

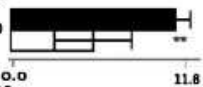

Verrucomicrobiae

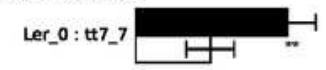

Ler_o : tt7

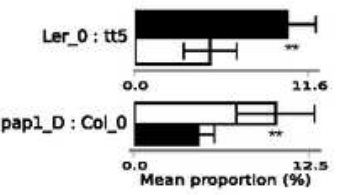

Thermoleophilia

Col_o : npr1_2

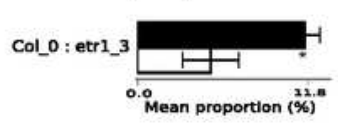

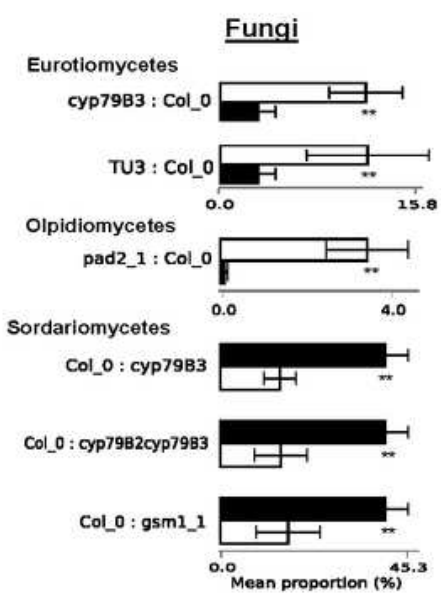

Eurotiomycetes

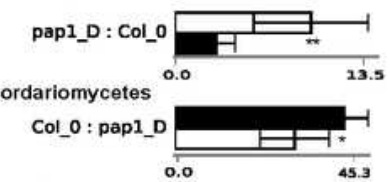

Tremellomycetes
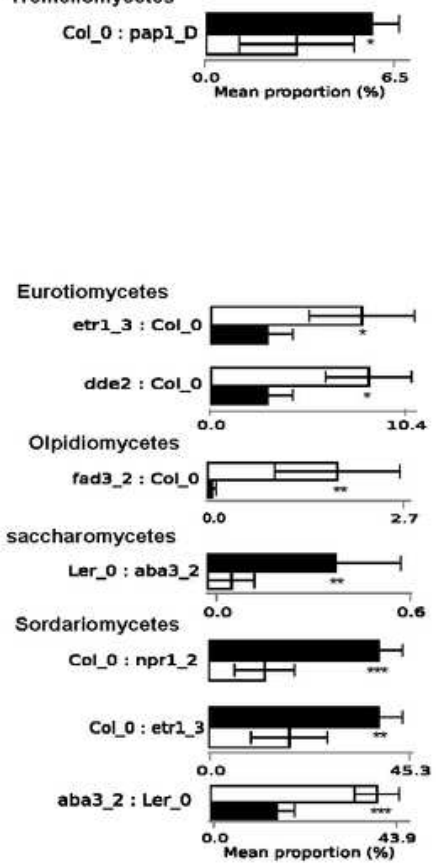

\section{Figure 1}

Bar plots showing mean distribution of reads assigned at class level in A) GLS mutants and their controls, B) FLV mutants and their controls and C) defense signaling mutants and their controls. Mutants were compared to their background controls by ANOVA, followed by the Tukey-Kramer post hoc test ( $p$ 
$<0.05)$ using the Benjamini and Hochberg (BH) FDR for multiple comparisons. Only top 10 taxa were used and taxa with small effect sizes were removed by filtering (effect size $=0.8$ ). The black and white bar plots represent controls and mutants respectively. Error bars represent standard deviations. The analysis was performed using the STAMP software (v2.1.3)
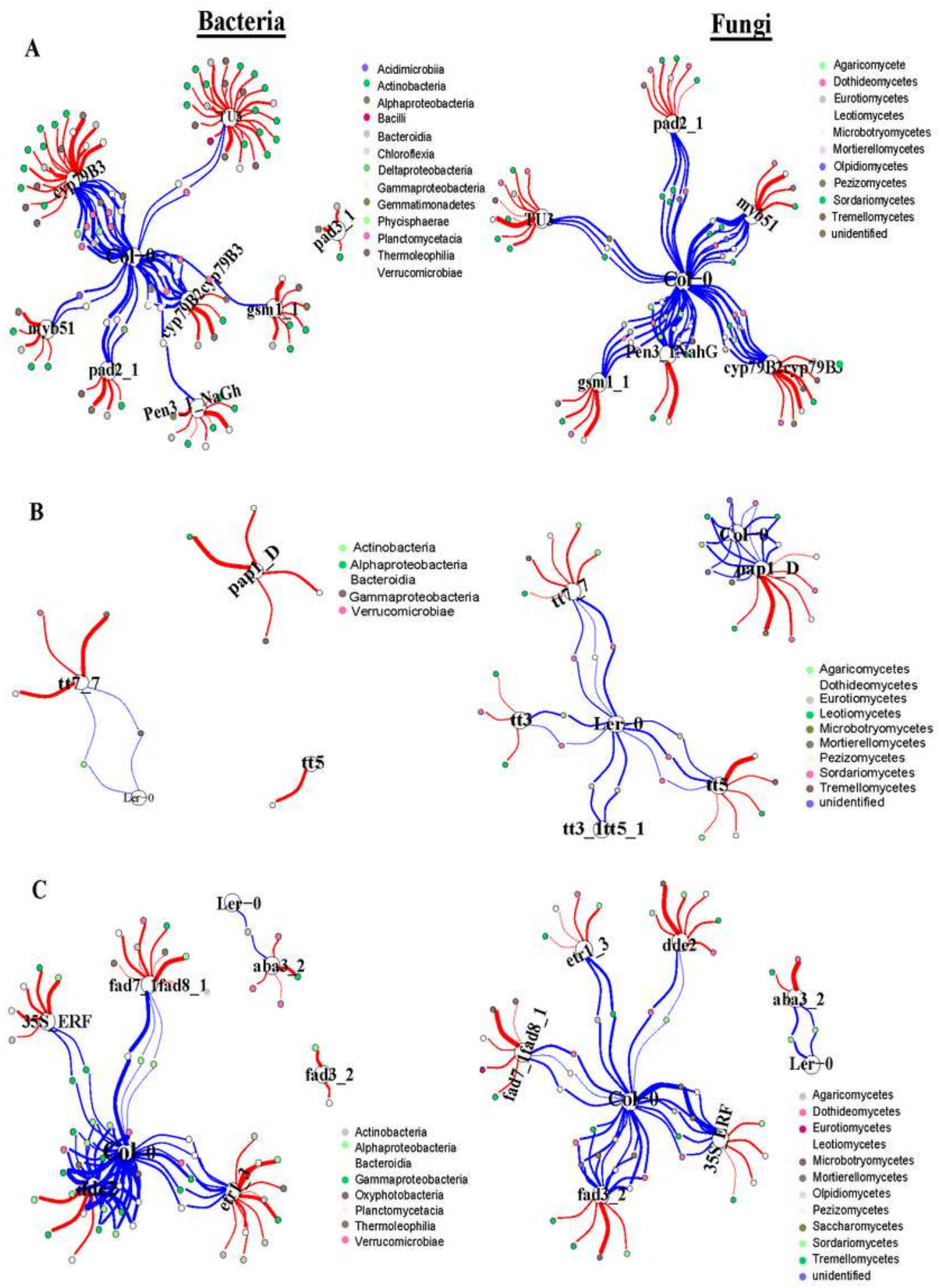

Figure 2 
Network showing microbial differential analysis between mutants and controls. Bacterial and fungal genera that were significantly affected are shown for A) GLS mutants and control. B) FLV mutants and control C) defense signaling mutants and control. Analysis performed using DESeq2. Enrichment in mutants are shown in red and in blue for controls. Line width indicates the significant level of effect.

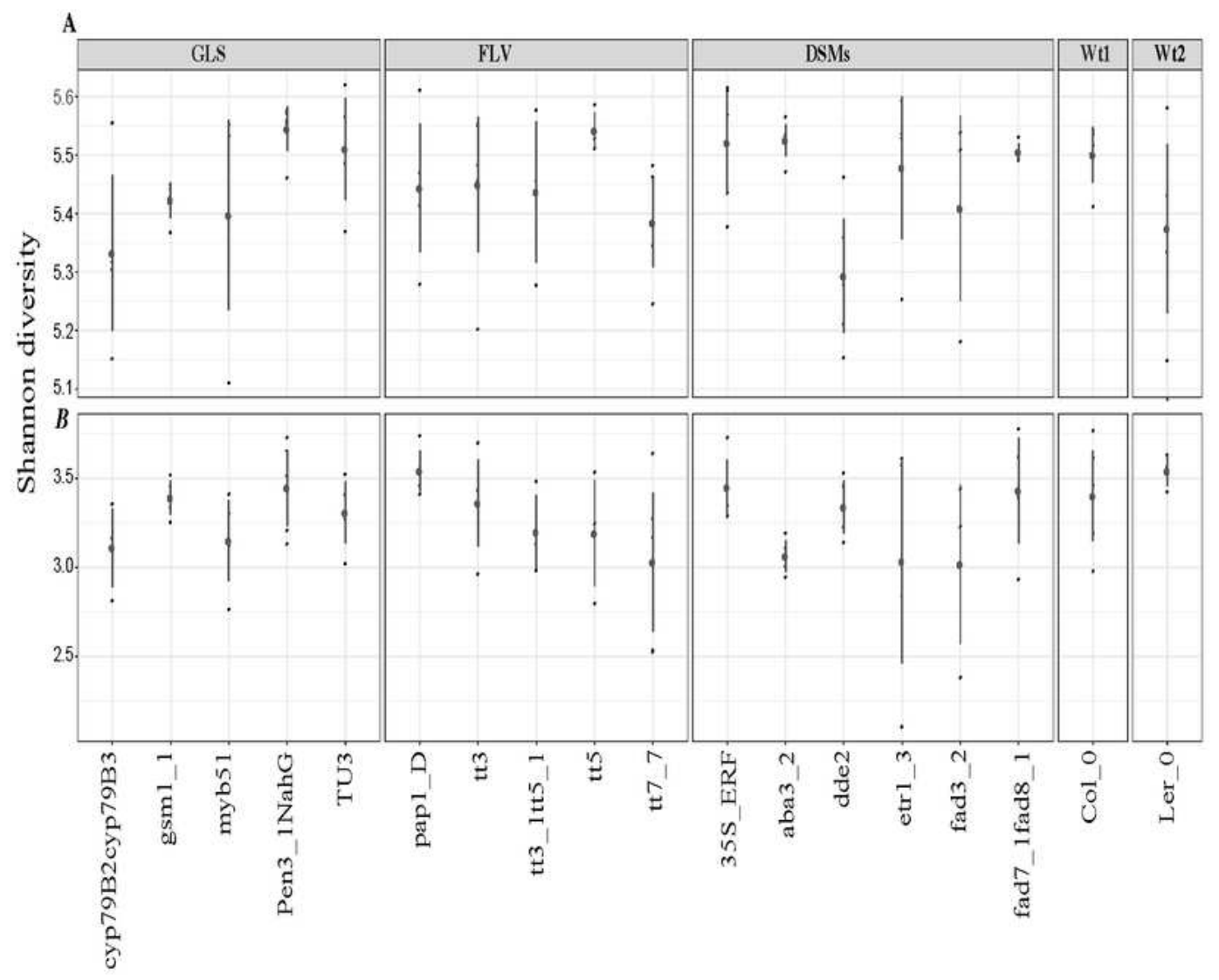

Figure 3 
Alpha diversity estimated using Shannon diversity for the respective genotypes. A) Bacterial and (B) fungal Shannon diversity in different genotypes. Samples with at least 3 replicates for both bacteria and fungi (with bacterial and fungal reads $>800$ and $>500$ respectively) are shown.
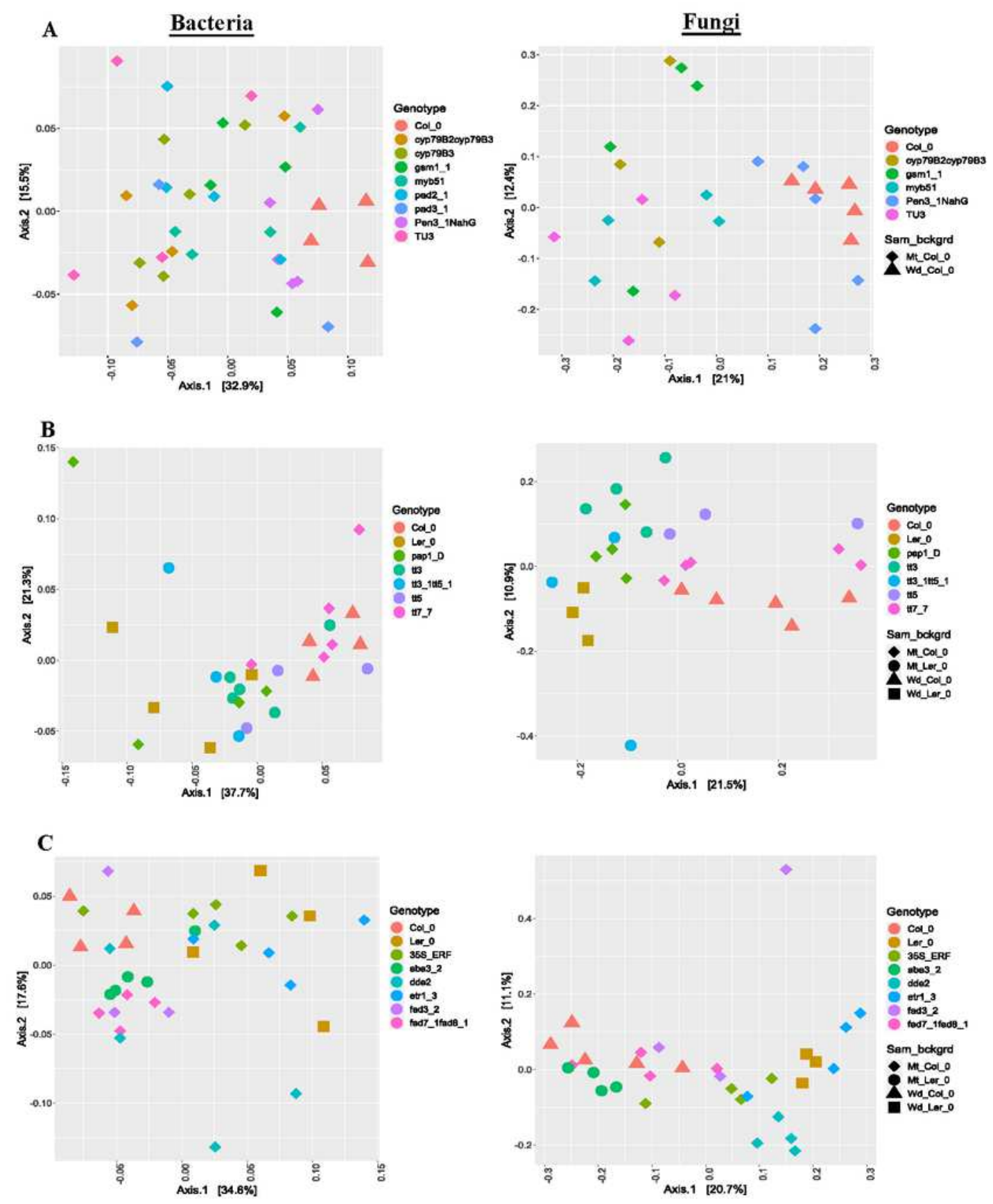

\section{Figure 4}

Principal coordinate analysis (PCoA) of weighted UniFrac distances (bacterial) and Bray-Curtis (fungal) between GLS (A), FLV (B) and DSM (C) genotypes. Individual genotypes and sample groups are shown in 
different colours and shapes.

\section{Supplementary Files}

This is a list of supplementary files associated with this preprint. Click to download.

- Additionalfile1.pdf

- ST1ListofArabidopsis.docx

- ST26BacFunDESeqoutput.xlsx 